CLINICAL STUDY

\title{
The post partum period and the onset of Graves' disease: an overestimated risk factor
}

\author{
Mario Rotondi, Barbara Pirali, Sara Lodigiani, Simona Bray, Paola Leporati, Spyridon Chytiris, Simona Balzano ${ }^{1}$ \\ Flavia Magri and Luca Chiovato \\ Unit of Internal Medicine and Endocrinology, Fondazione Salvatore Maugeri IRCCS, ISPESL Laboratory for Endocrine Disruptors, Chair of Endocrinology, \\ University of Pavia, Via S. Maugeri 10, I-27100 Pavia, Italy and ${ }^{1}$ Laboratory of Calculus and Quantitative Analysis, Department of Economics, University \\ of Cassino, I-03043 Cassino, Italy \\ (Correspondence should be addressed to L Chiovato; Email: luca.chiovato@fsm.it)
}

\begin{abstract}
Objective: Aggravation of autoimmune diseases due to a rebound reaction to the pregnancy-associated immune changes is common during the post partum (PP) period. Previous studies demonstrated that up to $45 \%$ of women developing Graves' disease (GD) in the childbearing age had a PP onset of disease. Thus, the PP period was identified as a major risk factor for GD onset.

Design: The aim of this study was to evaluate the role of the PP period as a risk factor for GD occurrence.

Methods: The reproductive histories of 291 consecutive GD patients (165 patients in the childbearing age and 126 in the non-childbearing age) were retrospectively collected.

Results: The rate of PP onset of GD in all patients with at least one successful pregnancy was 9.8 and $20.0 \%$ when only patients in the childbearing age were considered. In the entire cohort of GD women, independent of their age and parity status (i.e., the number of successful pregnancies), the rate of PP onset of GD was $7.2 \%$. The relative frequencies of the rate of $\mathrm{PP}$ onset of GD were similar in relation with increasing parity. The rates of false negative (nulliparous) and false positive (parous nonchildbearing + childbearing with a non-PP onset of GD) were estimated. The positive predictive value of the PP period for the onset of GD was less than $10 \%$.

Conclusions: The results of the current study would not support a role for the PP period as a major risk factor for de novo occurrence of GD.
\end{abstract}

European Journal of Endocrinology 159 161-165

\section{Introduction}

Normal pregnancy is associated with profound changes of the immune system $(1,2)$ and, among them, a shift to a type 2 polarized cytokine profile is necessary to minimize the maternal cell-mediated immune response against the fetus (3-5). These changes allow maintaining the fetalmaternal allograft, which is not rejected despite diverse paternal histocompatibility antigens (6-8). The pregnancy-induced alterations of the immune system largely influence the clinical activity of autoimmune diseases (9-11). With specific regard to autoimmune thyroid diseases (AITD), it is known that during pregnancy most patients experience a remission $(2,12)$, resulting from a downregulation of the Th1-mediated effector arms of the immune system. On the other hand, the post partum (PP) period is characterized by a rebound reaction to the pregnancy-associated immune suppression that accounts for the commonly observed aggravation of AITD during the puerperium $(13,14)$. Because thyroid autoimmunity primarily results from Th1 mechanisms, while circulating autoantibodies may be secondary to thyroid destruction, a Th2 to Th1 'return shift' in the puerperium would favor the outbreak of PP thyroiditis (PPT) $(14,15)$. Experimental and clinical evidence support the concept that PP AITD, including PPT and Graves' disease (GD), represent a prototype model for the aggravation of autoimmune diseases (14).

The prevalence of PP AITD was found to be largely variable among different studies $(16,17)$. In the only quantitative review of the entire literature on PP thyroid dysfunction, the pooled prevalence estimate weighted by sample size was $8.1 \%$ (18). Although PPT constitutes the most prevalent form of AITD developing in the PP period $(15,17,18)$, previous studies reported that, among all women developing GD in the childbearing age, $45 \%$ experienced the onset of disease in the PP period (19-22).

The aim of this study was to further evaluate the association between the PP period and the de novo occurrence of GD in an Italian population. The final goal was to define whether the PP period was indeed a major risk factor for the onset of GD, which might warrant screening and/or preventive strategies. 


\section{Patients and methods}

\section{Subjects}

In this study, data were retrospectively collected from all female patients with GD recruited in the outpatient clinic of the Unit of Internal Medicine and Endocrinology of the Fondazione S Maugeri IRCCS (Pavia, Italy) from 2002 to 2006.

In detail, 291 consecutive female patients who received a diagnosis of GD were enrolled. Their median age (and range) was 40 years (20-80 years). All patients were Caucasian, and lived in Pavia, Northern Italy, or in the surrounding area, a region with a mild iodine deficiency.

The diagnosis of hyperthyroid GD was established by measuring the serum concentrations of free thyroxine, free tri-iodothyronine, and thyrotrophin (TSH), and by searching for circulating thyroid antibodies: thyroglobulin antibodies, thyroperoxidase antibodies and TSH receptor antibodies (TRAbs). An ultrasound scan of the thyroid gland was performed in all cases, and was consistent with a diagnosis of GD, showing evidence of an increased thyroid blood flow. In order to differentiate Graves' hyperthyroidism from PPT with destructive thyrotoxicosis, evidence of increased thyroid blood flow at ultrasound and/or of increased radioiodine uptake was obtained in doubtful cases. Persistently positive tests for TRAb were also taken as supporting a diagnosis of GD. None of the patients had Graves' hyperthyroidism developing during gestation.

The number of successful pregnancies before the diagnosis of GD was recorded for all patients. Patients were considered as having a PP onset of GD when there was evidence for Graves' hyperthyroidism developing within 1 year after delivery.

In the present study, the childbearing age was defined taking into account the age of the youngest (20 years) and the oldest (43 years) patient experiencing a successful pregnancy. The group of patients developing GD in their childbearing age included 165 women, the remaining 126 patients developed $\mathrm{GD}$ in the nonchildbearing age. The parity status (i.e., the number of successful pregnancies) in the two groups of patients is shown in Table 1. The family history of the presence of AITD was also investigated. Informed consent, concerning the use of their clinical-pathological data for research purposes is routinely obtained from all patients attending our clinic. The present study was in accordance with the Institution Ethics Committee on human experimentation.

\section{Statistical design and analysis}

The statistical aim of this study was to estimate the strength of the PP period as a risk factor for the onset of GD. The study was carried out in an outpatient series of consecutive female patients with GD by retrospective
Table 1 Clinical characteristics of women receiving a diagnosis of Graves' disease in different periods of life.

\begin{tabular}{|c|c|c|c|c|}
\hline & & $\begin{array}{c}\text { Childbearing } \\
\text { age }\end{array}$ & $\begin{array}{c}\text { Non- } \\
\text { childbearing } \\
\text { age }\end{array}$ & $P$ value \\
\hline $\begin{array}{c}\text { Number of } \\
\text { cases }\end{array}$ & & 165 & 126 & \\
\hline $\begin{array}{l}\text { Age (years) } \\
\text { median } \\
\text { (range) }\end{array}$ & & $33(20-43)$ & $52(44-80)$ & $<0.0001$ \\
\hline $\begin{array}{l}\text { Successful } \\
\text { pregnancies, } \\
n(\%)\end{array}$ & $\begin{array}{l}0 \\
1 \\
2 \\
3 \\
\geq 4\end{array}$ & $\begin{array}{c}60(36.4 \%) \\
62(37.6 \%) \\
38(23.0 \%) \\
4(2.4 \%) \\
1(0.6 \%)\end{array}$ & $\begin{array}{c}16(12.7 \%) \\
52(41.3 \%) \\
44(34.9 \%) \\
8(6.3 \%) \\
6(4.8 \%)\end{array}$ & \\
\hline $\begin{array}{l}\text { Deliveries } \\
\quad(\text { mean } \pm \text { s.D. })\end{array}$ & & $1.47 \pm 0.66$ & $1.71 \pm 0.85$ & $<0.05^{\mathrm{a}}$ \\
\hline $\begin{array}{l}\text { Positive family } \\
\text { history for } \\
\text { AITD, } n(\%)\end{array}$ & & 37 (35.2\%) & 35 (31.8\%) & $N S^{a}$ \\
\hline
\end{tabular}

${ }^{a}$ Nulliparous patients were excluded from the analysis.

The parameters showing significant differences between the two groups are in italics.

collection of their reproductive history. Based upon the parity status at the moment of GD diagnosis, three subgroups were encountered: 1) nulliparous women developing GD, who represented the false-negative population, as they were never exposed to the risk factor to be tested (PP); 2) parous women developing GD after the PP period (more than 12 months after parturition), who represented the false-positive population; and 3) parous women developing GD in the PP period (within 12 months from parturition), who represented the true-positive population. The procedural approach to the evaluation of the PP period as a risk factor for the onset of GD was performed in several steps: i) estimation of the prevalence of GD in relation with the childbearing and the non-childbearing age; ii) analysis of the relative frequencies of GD developing in the PP or the non-PP period, in relation with the increasing number of successful pregnancies; and iii) estimation of the positive predictive value (PPV) of the PP period for developing GD, which was calculated as the number of true-positive cases/the number of true-positive + false-positive cases $\times 100$. Specifically, PPV is the number of women with at least one successful pregnancy showing a PP onset of GD/the number of women with at least one successful pregnancy with a PP onset of GD+the number of parous women with a non-PP onset of GD $\times 100$.

Statistical analysis was performed using the SPSS software (SPSS, Inc., Evanston, IL, USA). Between groups, comparisons were performed by means of Student's $t$-test for unpaired data and of Mann-Whitney $U$ test, according to a normal or a nonparametric distribution. Frequencies among groups were compared by $\chi^{2}$-test using Fisher's correction, when appropriate. A $P$ value $<0.05$ was considered statistically 
significant. Results are expressed as mean \pm s.D., unless otherwise stated.

\section{Results}

Data regarding the 291 GD patients, subdivided according to the occurrence of GD in the childbearing $(n=165)$ or in the non-childbearing age $(n=126)$, are shown in Table 1. After excluding, the nulliparous women who, for the purpose of this study, constituted the false-negative population, the two groups showed a difference for the mean number of successful pregnancies, which was significantly higher in women developing GD in the non-childbearing age. In our series of patients with at least one successful pregnancy, GD occurred in $105(48.8 \%)$ patients in the childbearing age and in $110(51.2 \%)$ patients after the childbearing age, with non-significant difference between the two groups. Similar rates for positive family history of AITD were found in patients developing GD in the childbearing age as compared with the non-childbearing age (35.2\% vs $31.8 \%$, respectively). The rate of positive family history for AITD, calculated in the entire study group $(n=291)$, was $37.8 \%$.

The influence of the PP period on the onset of GD was further evaluated by estimating the relative frequencies of GD developing in the PP or in the non-PP period, in relation to the total number of successful pregnancies (Fig. 1). The results show that the rate of occurrence of GD in the PP period decreased, even if statistical significance was not reached, from 13.1 to $5.1 \%$ in relation to increasing parity (from 1 to $>3$ successful pregnancies).

The rate of GD occurring in the PP period was $9.8 \%$ in the 215 women with at least one successful pregnancy, who constitute the entire cohort of patients exposed to the theoretical risk factor. As shown in Table 2, when the analysis was restricted to the 105 women in the childbearing age with at least one successful pregnancy, $21(20 \%)$ of them developed GD in the PP period.

The 21 women showing a PP onset of GD were significantly younger and had a higher, although not

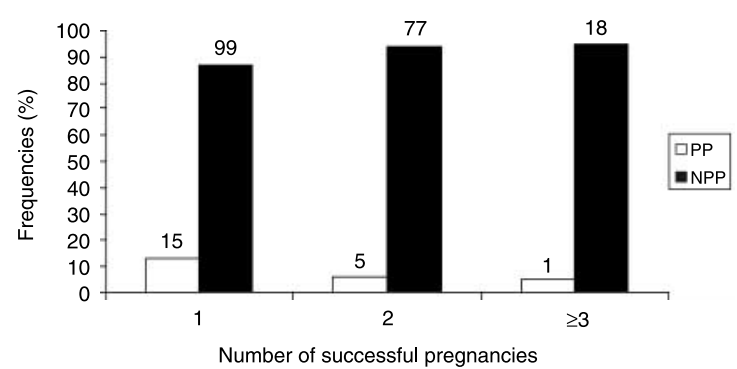

Figure 1 Relative frequencies of GD developing in the PP period or in the non-PP period in relation to the number of successful pregnancies. No significant difference in the rates of a PP onset of GD in relation to increasing parity was observed in patients with at least one successful pregnancy.
Table 2 Clinical characteristics of women in the childbearing age and at least one successful pregnancy, in relation to a post partum or a non-post partum onset of Graves' disease.

\begin{tabular}{lccc}
\hline & $\begin{array}{c}\text { Non-post } \\
\text { onset of GD }\end{array}$ & $\begin{array}{c}\text { of GD } \\
\text { partum onset } \\
\text { of }\end{array}$ & P value \\
\hline $\begin{array}{l}\text { Number of cases } \\
\text { Age (years) } \\
\text { median (range) }\end{array}$ & $21(20 \%)$ & $84(80 \%)$ & \\
$\begin{array}{c}\text { Deliveries } \\
\text { (mean } \pm \text { s.D.) }\end{array}$ & $1.33 \pm 0.57$ & $1.51 \pm 0.74$ & NS \\
$\begin{array}{c}\text { Positive family } \\
\text { history for AlTD, } \\
n(\%)\end{array}$ & $11(52.4 \%)$ & $26(30.9 \%)$ & NS \\
\hline
\end{tabular}

The parameters showing significant differences between the two groups are in italics.

significant, rate of positive family history for AITD, as compared with the 84 women showing a non-PP onset of GD. Furthermore, a similar number of successful pregnancies was found in women with GD, independently from the PP or the non-PP onset of the disease (Table 2).

The PPV of the PP period for developing GD, which was calculated according to the formula reported in

A Parous GD patients

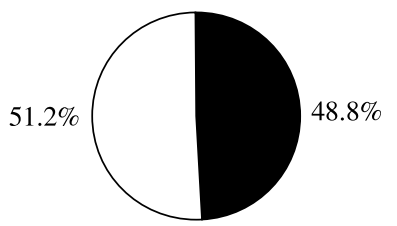

- Childbearing age $\square$ Non-childbearing age

B Childbearing aged GD patients

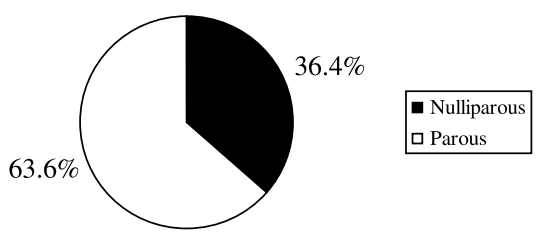

C Parous childbearing aged GD patients

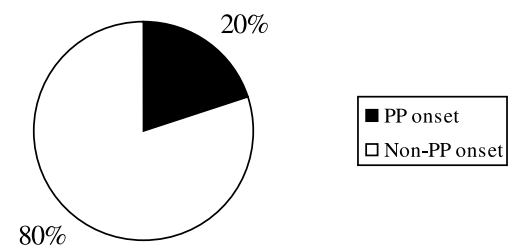

Figure 2 Occurrence of GD in subgroups of female patients: (A) parous women in childbearing and non-childbearing age. This subgroup encompasses all exposed patients, (B) patients in childbearing age who were either parous (exposed) or nulliparous (not exposed), and (C) parous patients in childbearing age with a PP (true positive) or non-PP (false positive) onset of GD. 
Statistical Methods, was 20.0 and $9.8 \%$ for women in the childbearing age and for the entire group of parous women respectively. Figure 2 schematically represents the prevalence of GD onset in our series of patients in relation with: a) childbearing and non-childbearing age, b) parity status, and c) PP or non-PP onset of the disease in exposed patients (parous women in childbearing age). The overall rate of GD showing a PP onset was as low as $7.2 \%(n=21)$, when the entire population of consecutive GD patients was taken into account (Fig. 3). In detail, GD onset was unrelated to the PP period in 126 patients developing the disease after their childbearing age; in 84 patients who were in their childbearing age and had completed at least one successful pregnancy; in 60 patients who were nulliparous (Fig. 3).

\section{Discussion}

The rate of $\mathrm{PP}$ onset of GD in our series was $9.8 \%$ in the entire cohort of patients exposed to the PP (at least one successful pregnancy) and $20.0 \%$ when the analysis was limited to patients in the childbearing age. It is important to note that the relative frequencies of patients with a PP onset of GD did not change in relation to increasing parity. Parous women in the childbearing age were divided in relation to a PP or a non-PP onset of GD. The two subgroups were similar for parity rate. However, women with a PP onset of GD were significantly younger and showed a higher, although not significant, rate of positive family history for AITD, as compared with those with a non-PP onset of GD. Despite the retrospective design of the current study not permitting addressing causality, the above-described findings would suggest a role for the PP period as a precipitating event for the onset of GD in genetically predisposed women, rather than a real causing factor. Overall, in our cohort of parous GD patients, the PPV of the PP period for the onset of GD was $9.8 \%$.

Our findings should be compared with previous reports indicating that, among parous women who
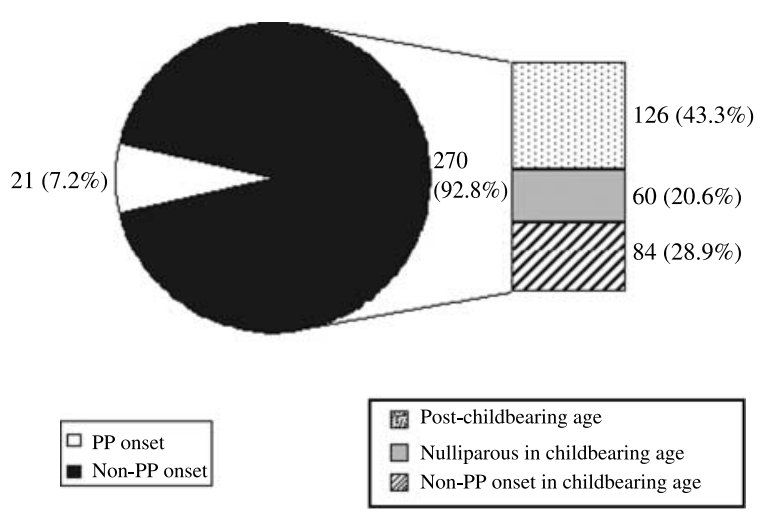

Figure 3 Occurrence of GD in the entire cohort of 291 female patients in relation with age, parity status, and PP or non-PP onset of the disease. develop GD in the childbearing age, as much as $45 \%$ of them did so in the PP period. These data lead to the conclusion that the PP period is a significant risk factor for the development of GD, at least in women of childbearing age (19-21). Our results do not confirm those previous observations and would not support a role for the PP period as a major risk factor for the onset of GD.

Several arguments may be considered in order to explain the discrepancy between the results of our study and those of our previous reports.

Firstly, all previous studies were performed in areas of iodine sufficiency or even moderately high iodine intake (Sweden, United States, and Japan). The much lower iodine intake of our Italian population might play a role in determining the different rate of women developing GD in the PP period. Secondly, ethnic differences should be taken into account when considering reports from Japan, indicating a high frequency of first trimester thyrotoxicosis (23) and of PP onset of GD (20). Thirdly, previous studies (19-21) included only women developing GD in their childbearing age. This might increase the weight of the PP period as a risk factor for the onset of GD, also in view of the fact that in our series nearly $50 \%$ of the entire population showed a non-childbearing onset of GD. Moreover, in our study, the percentage of patients developing GD in the $\mathrm{PP}$ period was $20 \%$ when only women in the childbearing age were considered, but fell to $9.8 \%$ when all parous women, representing the entire exposed population, were taken into account. Fourthly, at least for the earliest report, the use of insensitive methods for assaying TRAb and the lack of ultrasound data might be blamed as a possible reason for misclassifying patients with transient thyrotoxicosis due to PPT. This argument would be supported by the fact that, as stated by the authors themselves, some of their patients had a transient form of GD (19).

A fifth, and most likely explanation for the fact that, compared with previous studies, the strength of the PP period as a risk factor for the onset of GD was lower in our investigation, could be represented by the progressive reduction in the parity rate, which occurred in Italy over the last two decades. It is reasonable to estimate that a certain proportion of nulliparous women would have developed GD in the PP period if they had ever been pregnant. Furthermore, in our group of women of childbearing age, parous GD patients showed a lower mean number of successful pregnancies (1.3) as compared with that (2.0) reported in the studies by Jansson et al. (19) and Rochester et al. (21). Again, a higher number of successful pregnancies among parous GD patients might have increased the rate of PP onset of the disease.

The fact that nulliparous women accounted for a consistent proportion of GD patients in most investigated series, from $36.4 \%$ in this report to $61.8 \%$ in the study by Rochester et al. (21), deserves consideration. When 
analyzing the role of the PP period on the onset of GD, the exclusion of nulliparous patients (false-negative cases) is theoretically correct, but in order to draw conclusions for the general female population, it is important noting that more than one-third of women developing GD were never exposed to this potential risk factor. This observation further supports the concept that the PP period plays a minor role as a risk factor for the onset of GD, even in women of childbearing age. Indeed, as shown in Fig. 3, in a cohort of female Graves' patients, in which the onset of the disease was evaluated in relation to ages of life (childbearing-non-childbearing age) and parity status (parous-nulliparous), GD developing in the PP accounted for a proportion of all cases as low as $7.2 \%$. In the same cohort of female Graves' patients, a positive family history for AITD, which is commonly regarded as a weak risk factor for GD (22), was recorded in $37.8 \%$ of cases.

In conclusion, the results of this study demonstrate that: 1) the rate of PP onset of GD was as low as $7.2 \%$ in the entire cohort of consecutive female patients with GD, independently of their age and parity status; 2) the rate of PP onset of GD was low (20\%) even in parous women in the childbearing age; and 3) thus, the PP period cannot be considered a major risk factor for the onset of GD in the general female population.

\section{Acknowledgements}

The experiments reported in this paper were supported in part by funds from the Progetto di Ricerca Finalizzata ex art.12, del D.Lgs.502/92 - 2005.

\section{References}

1 Weetman AP. The immunology of pregnancy. Thyroid 19999 643-646.

2 Lazarus JH \& Kokandi A. Thyroid disease in relation to pregnancy: a decade of change. Clinical Endocrinology 200053 265-278.

3 Veenstra van Nieuwenhoven AL, Heineman MJ \& Faas MM. The immunology of successful pregnancy. Human Reproduction Update 20039 347-357.

4 Aagaard-Tillery KM, Silver R \& Dalton J. Immunology of normal pregnancy. Seminars in Fetal \& Neonatal Medicine 200611 279-295.

5 Piccinni MP. T-cell cytokines in pregnancy. American Journal of Reproductive Immunology $2002 \mathbf{4 7} 289-294$.

6 Lin H, Mosmann TR, Guilbert L, Tuntipopipat S \& Wegmann TG. Synthesis of $\mathrm{T}$ helper 2-type cytokines at the maternal-fetal interface. Journal of Immunology 1993151 4562-4573.
7 Piccinni MP, Beloni L, Livi C, Maggi E, Scarselli G \& Romagnani S. Defective production of both leukemia inhibitory factor and type 2 T-helper cytokines by decidual T cells in unexplained recurrent abortions. Nature Medicine 19984 1020-1024.

8 Moffett A \& Loke YW. The immunological paradox of pregnancy: a reappraisal. Placenta 200425 1-8.

9 Formby B. Immunologic response in pregnancy. Its role in endocrine disorders of pregnancy and influence on the course of maternal autoimmune diseases. Endocrinology and Metabolism Clinics of North America 199524 187-205.

10 Buyon JP, Nelson JL \& Lockshin MD. The effects of pregnancy on autoimmune diseases. Clinical Immunology and Immunopathology 199678 99-104.

11 Buyon JP. The effects of pregnancy on autoimmune diseases. Journal of Leukocyte Biology 199863 281-287.

12 Kung AWC \& Jones BM. A change from stimulatory to blocking antibody activity in Graves' disease during pregnancy. Journal of Clinical Endocrinology and Metabolism 199883 514-518.

13 Davies TF. The thyroid immunology of the postpartum period. Thyroid $19999675-684$.

14 Amino N, Tada H \& Hidaka Y. Postpartum autoimmune thyroid syndrome: a model of aggravation of autoimmune disease. Thyroid $19999705-713$.

15 Muller AF, Drexhage HA \& Berghout A. Postpartum thyroiditis and autoimmune thyroiditis in women of childbearing age: recent insights and consequences for antenatal and postnatal care. Endocrine Reviews 200122 605-630.

16 Walfish PG, Meyerson J, Provias JP, Vargas MT \& Papsin FR. Prevalence and characteristics of post-partum thyroid dysfunction: results of a survey from Toronto, Canada. Journal of Endocrinological Investigation 199215 265-272.

17 Shahbazian HB, Sarvghadi F \& Azizi F. Prevalence and characteristics of postpartum thyroid dysfunction in Tehran. European Journal of Endocrinology 2001145 397-401.

18 Nicholson WK, Robinson KA, Smallridge RC, Ladenson PW \& Powe NR. Prevalence of postpartum thyroid dysfunction: a quantitative review. Thyroid 200616 573-582.

19 Jansson R, Dahlberg PA, Winsa B, Meirik O, Safwenberg J \& Karlsson A. The post partum period constitutes an important risk for the development of clinical Graves' disease in young women. Acta Endocrinologica 1987116 321-325.

20 Tada H, Hidaka Y, Tsuruta E, Kashiwai T, Tamaki H, Iwatani Y \& Amino N. Prevalence of postpartum onset of disease within patients with Graves' disease of child-bearing age. Endocrine Journal 1994 41 325-327.

21 Rochester DB \& Davies TF. Increased risk of Graves' disease after pregnancy. Thyroid 200511 1287-1290.

22 Marinò M, Chiovato L \& Pinchera A. Graves' disease. In Endocrinology, edn 5, ch 100, pp 1995-2028. Eds LJ De Groot \& JL Jameson Saunders. Philadelphia Saunders Elsevier 2006.

23 Tagami T, Hagiwara H, Kimura T, Usui T, Shimatsu A \& Naruse M. The incidence of gestational hyperthyroidism and postpartum thyroiditis in treated patients with Graves' disease. Thyroid 2007 $17767-772$.

Received 22 April 2008

Accepted 8 May 2008 